To appear in

Dynamics of Continuous, Discrete and Impulsive Systems

http:monotone.uwaterloo.ca/ journal

\title{
Computation of an Over-Approximation of the Backward Reachable Set using Subsystem Level Set Functions
}

\author{
Dušan M. Stipanović ${ }^{1}$, Inseok Hwang ${ }^{1}$, and Claire J. Tomlin ${ }^{1}$ \\ ${ }^{1}$ Department of Aeronautics and Astronautics \\ Stanford University, Stanford, CA 94305
}

\begin{abstract}
In this paper, we present a method to decompose the problem of computing the backward reachable set for a dynamic system in a space of a given dimension, into a set of computational problems involving level set functions, each defined in a lower dimensional (subsystem) space. This allows the potential for great reduction in computation time. The overall system is considered as an interconnection of either disjoint or overlapping subsystems. The projection of the backward reachable set into the subsystem spaces is over-approximated by a level set of the corresponding subsystem level set function. It is shown how this method can be applied to two-player differential games. Finally, results of the computation of polytopic over-approximations of the unsafe set for the two aircraft conflict resolution problem are presented.
\end{abstract}

Keywords. Reachable sets; interconnected systems; over-approximations.

AMS (MOS) subject classification: 70H20, 49N70, 49N75, 93A14, 93A15

\section{Introduction}

Computation of reachable sets for dynamic systems has an important application in the automatic verification of safety properties and synthesis of safe controllers for air traffic systems $[12,18]$. The exact reachable set boundary 
is known to be the zero level set of the viscosity solution [2] of a HamiltonJacobi type of partial differential equation (PDE) [14]. To the best of our knowledge, Leitmann was the first to recognize the relationship between Bellman functions and the boundaries of the reachable sets [11]. In [22] it was shown how to approximate boundaries of reachable sets with an arbitrary accuracy using smooth functions. In the sequence of papers by Khrustalev $[4,5]$, locally Lipschitz functions are used to describe, arbitrarily accurate, under- and over-approximations of reachable sets. Finally, polytopic [19] and ellipsoidal approximations $[7,8,9,10]$ were developed to approximate reachable sets for linear systems (with and without perturbations).

The numerical solutions which provide convergent approximations of reachable sets for dynamic systems have computational complexity which is exponential in the continuous variable dimension $[14,13]$. To overcome this problem, in [3] the authors use a polytopic approximation method [19], based on optimal control methods (e.g., $[6,20])$, and polytopic level set functions, to efficiently compute approximations of forward and backward reachable sets for linear dynamic systems. This approach is extended to feedback linearizable nonlinear systems, linear dynamic games, and norm-bounded nonlinear systems in [3].

In [21], Vincent and Wu consider an approximation of the projection of the backward reachable (controllable) set for a class of hierarchical dynamic systems. Mitchell and Tomlin [15] approximate the backward reachable set for differential games by solving a set of lower dimensional Hamilton-JacobiIsaacs PDEs in projected spaces.

In this paper, we propose a method which decomposes the overall problem of computing an over-approximation for the backward reachable set of a general nonlinear dynamic system, into a number of lower dimensional computational problems. By doing so, the computational complexity of the problem is decreased: in general, we solve many reachability problems of lower dimension than the original reachability problem. For implementation, com- 
Comp. of an Over-App. of the Back. Reach. Set using Sub. Level Set Func. 3

putational load may be distributed over a network of parallel computers. The overall dynamic system is considered as a set of interconnected subsystems. Then, with each subsystem we associate a level set function of Lipschitz type of which a particular level set is an over-approximation of the boundary of the backward reachable set in the subsystem state space (projection space). The interconnection of these sets is an over-approximation of the backward reachable set. The idea for this approach has been strongly motivated by the successful application of vector Liapunov functions to establish stability of interconnected dynamic systems [23].

The paper is organized as follows. In Section 2 we present the mathematical background for backward reachable sets. The analysis of the computation of over-approximations in the subsystem (projected) spaces is provided in Section 3. The method is extended to differential games in Section 4, with an application to the computation of the unsafe set in the two aircraft collision avoidance problem. Conclusions are presented in Section 5.

\section{Backward Reachable (Controllable) Sets}

Let us consider the following dynamic system

$$
\dot{x}=f(t, x, u), \quad t \in \mathbf{T}=\left[0, t_{f}\right], x\left(t_{f}\right) \in \mathbf{X}_{f}
$$

where $0 \leq t_{f}<\infty, x \in \mathbb{R}^{n}$ is the state, $u \in \mathbf{U} \subset \mathbb{R}^{m}$ is the control input with $\mathbf{U}$ a compact set independent of the state $x$. A compact set of final states is denoted as $\mathbf{X}_{f}, \mathbf{X}_{f} \subset \mathbb{R}^{n}$. The function $f: \mathbf{T} \times \mathbb{R}^{n} \times \mathbf{U} \rightarrow \mathbb{R}^{n}$ is assumed to be Lipschitz continuous. The control input function $t \rightarrow u(t)$ is a measurable function, and the trajectory of the system $t \rightarrow x(t)$ is an absolute continuous function such that equation (1) is satisfied almost everywhere. We will refer to the set of final states as the target set.

The backward reachable (controllable) set (from a given target set $\mathbf{X}_{f}$ ) for the system (1) is defined as follows: 
Definition 1. The backward reachable set $\mathbb{C}(t)$ at time $t(t \in \boldsymbol{T})$, of the system (1) from the target set $\boldsymbol{X}_{f}$, is the set of all states $x$ for which there exists an admissible control input $u(\tau)\left(\tau \in\left[t, t_{f}\right]\right)$ and a corresponding absolute continuous trajectory $x(\tau)\left(\tau \in\left[t, t_{f}\right]\right)$ of the system (1), such that $x\left(t_{f}\right) \in \boldsymbol{X}_{f}$ and $x=x(t)$.

We define the Hamiltonian for the backward propagation of the system (1) as

$$
H_{f}(t, x, p)=\min _{u \in \mathbf{U}}\left\{p^{T} f(t, x, u)\right\}
$$

with $p \in \mathbb{R}^{n}$ being the adjoint state vector. The inner product of two vectors $a, b \in \mathbb{R}^{n}$, is denoted as $a^{T} b$. It is well known [14] that for any $t \in \mathbf{T}$, the exact boundary of the backward reachable set can be computed as the zero level set of the viscosity solution $v(t, x)$ of the Hamilton-Jacobi equation,

$$
\frac{\partial v}{\partial t}+H_{f}\left(t, x, \frac{\partial v}{\partial x}\right)=0
$$

with the final condition $v\left(t_{f}, x\right)$ having zero level set which is the boundary of the target set $\mathbf{X}_{f}$. Let us rewrite system (1) in decomposed form as a set of $N$ subsystems

$$
\dot{x}_{i}=f_{i}(t, x, u), \quad i \in \mathbf{N}, \quad \mathbf{N}=\{1, \ldots, N\}
$$

where $x_{i} \in \mathbb{R}^{n_{i}}$ is the $i$-th subsystem state such that $x=\left[x_{1}^{T}, \ldots, x_{N}^{T}\right]^{T} \in \mathbb{R}^{n}$, that is, the subsystems are disjoint. Then,

$$
\begin{aligned}
H_{f}(t, x, p) & =\min _{u \in \mathbf{U}}\left\{p^{T} f(t, x, u)\right\} \\
& =\min _{u \in \mathbf{U}}\left\{\sum_{i=1}^{N} p_{i}^{T} f_{i}(t, x, u)\right\} \geq \sum_{i=1}^{N} \min _{u \in \mathbf{U}}\left\{p_{i}^{T} f_{i}(t, x, u)\right\}
\end{aligned}
$$

By defining the subsystem Hamiltonians as

$$
H_{f_{i}}\left(t, x, p_{i}\right)=\min _{u \in \mathbf{U}}\left\{p_{i}^{T} f_{i}(t, x, u)\right\}
$$

from equations (5) and (6) it follows that

$$
H_{f}(t, x, p) \geq \sum_{i=1}^{N} H_{f_{i}}\left(t, x, p_{i}\right)
$$


Comp. of an Over-App. of the Back. Reach. Set using Sub. Level Set Func. 5

If we assume that each subsystem has its own independent input, that is,

$$
f_{i}(t, x, u)=\hat{f}_{i}\left(t, x, u_{i}\right)
$$

with $u_{i} \in \mathbb{R}^{m_{i}}, m=\sum_{i=1}^{N} m_{i}$, (that is, decomposition of the input vector is disjoint) then using (5), (6), and (8) we obtain the following:

$$
H_{f}(t, x, p)=\sum_{i=1}^{N} H_{\hat{f}_{i}}\left(t, x, p_{i}\right)
$$

Thus, when the subsystems can be chosen to have independent inputs, inequality (7) becomes equality (9).

To conclude this section, let us state that the motivation for deriving inequality (7) and equality (9) is the idea of converting the high dimensional problem of computing the backward reachable set for the overall system into a set of lower dimensional problems involving subsystem Hamiltonians.

\section{Over-Approximations of the Subsystem Pro- jections of the Backward Reachable Sets}

In order to compute an over-approximation of the backward reachable set, denoted as $\Re(t),(\mathbb{C}(t) \subseteq \Re(t))$, to each subsystem we associate a level set function $\left(t, x_{i}\right) \rightarrow v_{i}\left(t, x_{i}\right)$ which is assumed to be Lipschitz continuous with positive Lipschitz constant $k_{i}$. By computing its derivative along the solution of system (1) we obtain

$$
\left.\frac{d v_{i}\left(t, x_{i}\right)}{d t}\right|_{(1)}=\frac{\partial v_{i}}{\partial t}+\left(\frac{\partial v_{i}}{\partial x}\right)^{T} f(t, x, u)=\frac{\partial v_{i}}{\partial t}+\left(\frac{\partial v_{i}}{\partial x_{i}}\right)^{T} f_{i}(t, x, u)
$$

For $p_{i}=\partial v_{i} / \partial x_{i}$, from equation (6) we obtain

$$
\left(\frac{\partial v_{i}}{\partial x_{i}}\right)^{T} f_{i}(t, x, u) \geq H_{f_{i}}\left(t, x, \frac{\partial v_{i}}{\partial x_{i}}\right)
$$

Now, from equations (10) and (11) it follows:

$$
\left.\frac{d v_{i}}{d t}\right|_{(1)} \geq \frac{\partial v_{i}}{\partial t}+H_{f_{i}}\left(t, x, \frac{\partial v_{i}}{\partial x_{i}}\right)
$$


If there exists a measurable function $\mu_{i}(t)$ such that (see $[4,5,8]$ for application to a single system)

$$
\frac{\partial v_{i}}{\partial t}+H_{f_{i}}\left(t, x, \frac{\partial v_{i}}{\partial x_{i}}\right) \geq \mu_{i}(t)
$$

for all $t \in \mathbf{T}$ and $x \in \mathbb{R}^{n}$, then from (12) and (13) it follows:

$$
v_{i}\left(t, x_{i}(t)\right) \leq-\int_{t}^{t_{f}} \mu_{i}(\tau) d \tau+\max _{x_{i}\left(t_{f}\right) \in\left(\mathbf{X}_{f}\right)_{i}}\left\{v_{i}\left(t_{f}, x_{i}\left(t_{f}\right)\right)\right\}
$$

where $\left(\mathbf{X}_{f}\right)_{i}$ is the projection of the target set into the subsystem space $\mathbb{R}^{n_{i}}$, where $x_{i} \in \mathbb{R}^{n_{i}}$. It is important to note that the choice of an appropriate $\mu_{i}(t)$ in inequality (13) is very much problem dependent, and no suggestions of how to compute the set of measurable functions $\left\{\mu_{i}(t)\right\}_{i=1}^{N}$ for the general system can be given. We will address this issue in more detail in Section 3.1 by considering interconnected systems as an example of systems with a special structure.

The over-approximation of the reachable set for the subsystem with state $x_{i}$ is given by the following formula $[4,5,8]$ :

$$
\Re_{i}(t)=\left\{x_{i} \mid v_{i}\left(t, x_{i}\right) \leq-\int_{t}^{t_{f}} \mu_{i}(\tau) d \tau+\max _{x_{i}\left(t_{f}\right) \in\left(\mathbf{X}_{f}\right)_{i}}\left\{v_{i}\left(t_{f}, x_{i}\left(t_{f}\right)\right)\right\}\right\}
$$

Notice that from equation (15) it follows that we can compute an overapproximation of the backward reachable set for only a portion of the state

space, that is, $x_{i}$. The over-approximation of the reachable set for the overall state is given by

$$
\Re(t)=\Re_{1}(t) \times \ldots \times \Re_{N}(t), \mathbb{C}(t) \subseteq \Re(t)
$$

where symbol " $\times$ " denotes the Cartesian product.

\subsection{Interconnected Systems}

In this section we consider a class of nonlinear systems with a special structure, that is, interconnected systems, for which we can analyze the procedure 
Comp. of an Over-App. of the Back. Reach. Set using Sub. Level Set Func. 7

of determining a bound, represented by inequality (13), in more detail. Let us assume that functions $\left\{f_{i}\right\}_{i=1}^{N}$ in equation (4) are of the following form:

$$
f_{i}(t, x, u)=g_{i}\left(t, x_{i}, u\right)+h_{i}(t, x, u)
$$

where $g_{i}: \mathbf{T} \times \mathbb{R}^{n_{i}} \times \mathbf{U} \rightarrow \mathbb{R}^{n_{i}}$ is a Lipschitz continuous function that represents subsystem dynamics, and $h_{i}: \mathbf{T} \times \mathbb{R}^{n} \times \mathbf{U} \rightarrow \mathbb{R}^{n_{i}}$ is a Lipschitz continuous function that represents interconnections between subsystems, for all $i, i \in \mathbf{N}$.

Let us assume that for each $i$ there is a measurable function $t \rightarrow \mu_{x_{i}}(t)$ over the finite time horizon $\mathbf{T}$, such that

$$
\frac{\partial v_{i}}{\partial t}+H_{g_{i}}\left(t, x_{i}(t), \frac{\partial v_{i}}{\partial x_{i}}\right) \geq \mu_{x_{i}}(t)
$$

where the subsystem Hamiltonian $H_{g_{i}}(\cdot, \cdot, \cdot)$ (as defined in (6)) is computed with respect to the function $g_{i}(\cdot, \cdot, \cdot)$ defined in (17). Then,

$$
\begin{aligned}
\left.\frac{d v_{i}\left(t, x_{i}\right)}{d t}\right|_{(1)} & =\frac{\partial v_{i}}{\partial t}+\left(\frac{\partial v_{i}}{\partial x_{i}}\right)^{T} f_{i}(t, x, u) \geq \frac{\partial v_{i}}{\partial t}+H_{f_{i}}\left(t, x, \frac{\partial v_{i}}{\partial x_{i}}\right) \\
& \geq \frac{\partial v_{i}}{\partial t}+H_{g_{i}}\left(t, x, \frac{\partial v_{i}}{\partial x_{i}}\right)+\min _{u \in \mathbf{U}}\left\{\left(\frac{\partial v_{i}}{\partial x_{i}}\right)^{T} h_{i}(t, x, u)\right\} \\
& \geq \mu_{x_{i}}(t)+\min _{u \in \mathbf{U}}\left\{\left(\frac{\partial v_{i}}{\partial x_{i}}\right)^{T} h_{i}(t, x, u)\right\}
\end{aligned}
$$

We assume that the interconnections satisfy sector bounds [23]

$$
\left.\| h_{i}(t, x, u)\right\} \| \leq \sum_{j=1}^{N} \beta_{i j} \mu_{x_{j}}(t)
$$

for some $\beta_{i j}$ 's. Since $v_{i}\left(t, x_{i}\right)$ is a Lipschitz function in $x_{i}$ with positive Lipschitz constant $k_{i}$, we define

$$
\beta_{i}(t)=\sum_{j=1}^{N} k_{i} \beta_{i j} \mu_{x_{j}}(t)
$$

such that

$$
\min _{u \in \mathbf{U}}\left\{\left(\frac{\partial v_{i}}{\partial x_{i}}\right)^{T} h_{i}(t, x, u)\right\} \geq-\beta_{i}(t)
$$

From (19) and (22) it follows that:

$$
\left.\frac{d v_{i}\left(t, x_{i}\right)}{d t}\right|_{(1)} \geq \mu_{x_{i}}(t)-\beta_{i}(t)
$$


By equating $\mu_{i}(t)=\mu_{x_{i}}(t)-\beta_{i}(t)$, and integrating (23), the over-approximation of the backward reachable set for $x_{i}$ is given by

$$
\Re_{i}(t)=\left\{x_{i} \mid v_{i}\left(t, x_{i}\right) \leq-\int_{t}^{t_{f}} \mu_{i}(\tau) d \tau+\max _{x_{i}\left(t_{f}\right) \in\left(\mathbf{X}_{f}\right)_{i}}\left\{v_{i}\left(t_{f}, x_{i}\left(t_{f}\right)\right)\right\}\right\}
$$

which has the same form as (15), and the over-approximation $\Re(t)$ of the backward reachable set for the state $x$ is obtained using (16).

\subsection{Overlapping Over-Approximations}

We have considered decomposing the overall state into disjoint subsystems. In this section, we allow partitioned subsystem states to overlap (for more details on the overlapping subsystems see [23]). Consider equations (14) and (15), and for simplicity of the presentation (and without loss of generality), assume that we have only two subsystems with states $x_{1} \in \mathbb{R}^{n_{1}}$, and $x_{2} \in$ $\mathbb{R}^{n_{2}}$, such that they overlap, that is, $x_{1}$ and $x_{2}$ share components, meaning $x_{1} \cap x_{2}=x_{12}, x_{12} \in \mathbb{R}^{n_{12}}, n_{12} \geq 1$. Notice that $n_{1}+n_{2}-n_{12}=n$. We define extended over-approximations as

$$
\begin{aligned}
& \bar{\Re}_{1}(t)=\Re_{1}(t) \times \underbrace{\mathbb{R} \times \ldots \times \mathbb{R}}_{n-n_{1} \text { times }} \\
& \bar{\Re}_{2}(t)=\underbrace{\mathbb{R} \times \ldots \times \mathbb{R} \times \Re_{2}(t)}_{n-n_{2} \text { times }}
\end{aligned}
$$

where $\Re_{1}(t)$ and $\Re_{2}(t)$ are computed as in (15) or, in the case of interconnected systems, as in (24). Then, the following is true:

$$
\left(\mathbb{C}(t) \subseteq \bar{\Re}_{1}(t)\right) \wedge\left(\mathbb{C}(t) \subseteq \bar{\Re}_{2}(t)\right) \Rightarrow \mathbb{C}(t) \subseteq \bar{\Re}_{1}(t) \cap \bar{\Re}_{2}(t)
$$

where symbol " $\wedge$ " denotes the "and" logic operator. In other words, we can compute an over-approximation as an intersection of any set of extended lower dimensional over-approximations in the subspaces that cover the whole space. The extension to $N$ subsystems with overlapping states is clear. This gives us more freedom, since we are not any more restricted only to disjoint partitions of the state space. 
Comp. of an Over-App. of the Back. Reach. Set using Sub. Level Set Func. 9

\section{Differential Games with Application to Con- flict Resolution}

In this section, we show how the analysis presented in Section 3 carries over to accommodate the computation of the backward reachable set for the twoplayer differential game. Let us consider a two-player game described as the following dynamic system:

$$
\dot{x}_{i}=g_{i}\left(x_{i}\right)+q_{i 1}(x) u+q_{i 2}(x) d+h_{i}(x), \quad t \in \mathbf{T}, i \in \mathbf{N}
$$

where functions $u=u(\cdot) \in U=\{$ measurable functions : $\mathbf{T} \rightarrow \mathbf{U}\}$ and $d=d(\cdot) \in D=\{$ measurable functions $: \mathbf{T} \rightarrow \mathbf{D}\}$ are the control functions of the first and the second player, respectively [1]. We assume that $\mathbf{U}, \mathbf{U} \subset \mathbb{R}^{m_{u}}$, and $\mathbf{D}, \mathbf{D} \subset \mathbb{R}^{m_{d}}$ are compact sets. The right-hand side of equation (27) is Lipschitz continuous, and the solution trajectories $t \rightarrow x(t)$ are assumed to be absolute continuous. The partition $\left\{x_{i}\right\}_{i=1}^{N}$ of the state $x$ is a set of subsystem states that are either disjoint or overlapping. The target set is denoted as $\mathbf{X}_{f}$.

In order to define a game, let us introduce the nonanticipating strategy for the first player as [1]:

Definition 2. A strategy for the first player, which is a map $\alpha: D \rightarrow U$, is nonanticipating if for any $t>0$ and $d, \tilde{d} \in D, d(\tau)=\tilde{d}(\tau)$ for all $\tau \leq t$ implies $\alpha[d](\tau)=\alpha[\tilde{d}](\tau)$ for all $\tau \leq t$.

We denote the set of nonanticipating strategies for the first player as $\Gamma$, and recall that the feedback strategy $\alpha[d](t) \triangleq \phi(t, x(t))$, where $\phi: \mathbf{T} \times \mathbb{R}^{n} \rightarrow$ $\mathbf{U}$ such that $t \rightarrow \phi(t, x(t))$ is a measurable function along the solutions of (27), and the solutions are unique for a given initial condition, is nonanticipating [1].

Now, we can define the backward reachable set for the system (27) as follows [14]: 
Definition 3. The backward reachable set $\mathbb{C}(t)$ at time $t(t \in \boldsymbol{T})$, of the system (27) from the target set $\boldsymbol{X}_{f}$, is the set of all states $x$ for which there exists a nonanticipating strategy $\alpha \in \Gamma$ for the first player such that for any admissible control input $d \in D$, an absolute continuous trajectory $x(\tau)$ $\left(\tau \in\left[t, t_{f}\right]\right)$ of the system (27) satisfies $x\left(t_{f}\right) \in \boldsymbol{X}_{f}$ and $x=x(t)$.

The interpretation of Definition 3 is that there exists a strategy for the first player that drives the system to $\mathbf{X}_{f}$ despite the choice of the control input of the second player.

To compute an approximation (at this point it is important to note that this is not necessarily an over-approximation for the backward reachable set as defined in Definition 3), for a given Lipschitz level set function $\left(t, x_{i}\right) \rightarrow$ $v\left(t, x_{i}\right)$ we introduce

$$
\begin{aligned}
\lambda_{i}(t, x)= & \frac{\partial v_{i}}{\partial t}+\left(\frac{\partial v_{i}}{\partial x}\right)^{T} g_{i}\left(x_{i}\right)+\left(\frac{\partial v_{i}}{\partial x_{i}}\right)^{T} q_{i 1}(x) \tilde{u}(t, x) \\
& +\left(\frac{\partial v_{i}}{\partial x_{i}}\right)^{T} q_{i 2}(x) \tilde{d}(t, x)+\beta_{i}(t)
\end{aligned}
$$

where

$$
\begin{aligned}
& \tilde{u}(t, x)=\underset{u \in \mathbf{U}}{\arg \min }\left\{\left(\frac{\partial v_{i}\left(t, x_{i}\right)}{\partial x_{i}}\right)^{T} q_{i 1}(x) u\right\} \\
& \tilde{d}(t, x)=\underset{d \in \mathbf{D}}{\arg \max }\left\{\left(\frac{\partial v_{i}\left(t, x_{i}\right)}{\partial x_{i}}\right)^{T} q_{i 2}(x) d\right\}
\end{aligned}
$$

and we assume that $\left(\partial v_{i} / \partial x_{i}\right)^{T} h_{i}(x(t)) \leq \beta_{i}(t)$ holds for all $t \in \mathbf{T}$ and $x \in \mathbb{R}^{n}$. If there exist a measurable function $\mu_{i}(t)$ such that

$$
\mu_{i}(t) \leq \lambda_{i}(t, x)
$$

for all $t \in \mathbf{T}$ and $x \in \mathbb{R}^{n}$, then the following set is the approximation of the backward reachable projection into $\mathbb{R}^{n_{i}}$, the state space of the subsystem with state $x_{i}$ :

$$
\Re_{i}(t)=\left\{x_{i} \mid v_{i}\left(t, x_{i}\right) \leq-\int_{t}^{t_{f}} \mu_{i}(\tau) d \tau+\max _{x_{i}\left(t_{f}\right) \in\left(\mathbf{X}_{f}\right)_{i}}\left\{v_{i}\left(t_{f}, x_{i}\left(t_{f}\right)\right)\right\}\right\}
$$

Notice that in $(29), \tilde{d}(t, x)$ is a function of both independent variable $t$ and dependent variable $x$ and thus does not belong to the set of measurable 
Comp. of an Over-App. of the Back. Reach. Set using Sub. Level Set Func. 11

functions $D$. In the case when $\tilde{d}(t, x) \equiv \tilde{d}(t)$, equation (31) does represent an over-approximation of the reachable set as defined in Definition 3. In general, since the set of feedback strategies is larger then the set $D$ this might not be true. The motivation to compute an approximation using equations (28)-(31) is that it is computationally efficient, and that in a number of important applications the maximization of the control strategies for the second player does depend only on $t$ (as it is the case in which we use polytopic approximations for the linear two-player differential games as considered in the conflict resolution problem).

\subsection{Conflict Resolution between Two Aircraft}

To demonstrate the proposed procedure we consider the two aircraft collision avoidance problem [14] which is modeled as differential game (27). In Figure 1 we show the relative configuration of the two aircraft where aircraft 1 tries to avoid the collision regardless of the behavior of aircraft 2 . In this problem we wish to compute the backward reachable (unsafe) set from the target set (protected zone), that is, the set which includes all the states (in relative coordinates) from which aircraft 2 can choose a control that will lead to loss of separation for any control strategy of aircraft 1 .

We use the planar kinematic model for each aircraft, and after dynamic extension and feedback linearization of the model [16], we obtain the following linear model in terms of relative coordinates of the two aircraft [17]:

$$
\dot{x}=\left[\begin{array}{cccc}
0 & 0 & 1 & 0 \\
0 & 0 & 0 & 1 \\
0 & 0 & 0 & 0 \\
0 & 0 & 0 & 0
\end{array}\right] x+\left[\begin{array}{cc}
0 & 0 \\
0 & 0 \\
1 & 0 \\
0 & 1
\end{array}\right] u_{2}-\left[\begin{array}{cc}
0 & 0 \\
0 & 0 \\
1 & 0 \\
0 & 1
\end{array}\right] u_{1}
$$

where $x \in \mathbb{R}^{4}$ is the state vector, $u_{1} \in \mathbf{U}_{1} \subset \mathbb{R}^{2}$, and $u_{2} \in \mathbf{U}_{2} \subset \mathbb{R}^{2}$, are control inputs of the two aircraft, respectively. From the definition of the unsafe set notice that $u_{1}$ corresponds to $d$, and $u_{2}$ corresponds to $u$ in 


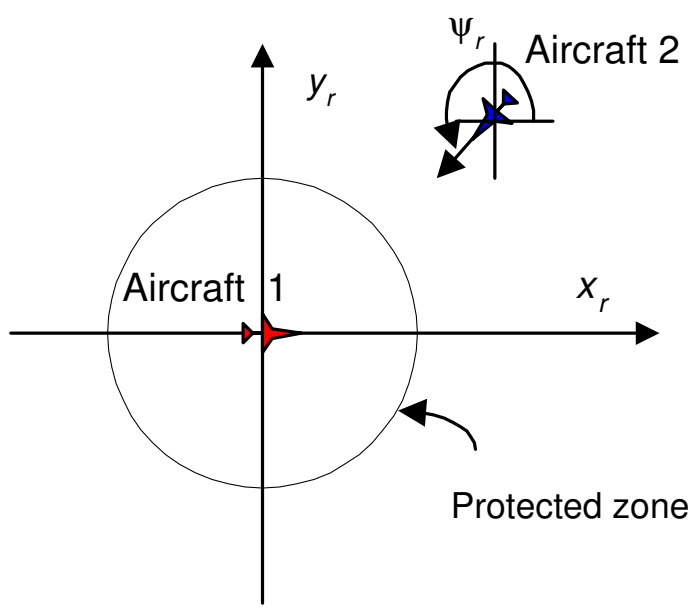

Figure 1: Relative coordinate system for the two aircraft conflict resolution problem.

equation (27). We decompose the state vector $x$ as $x=\left[x_{1}^{T}, x_{2}^{T}\right]^{T}$, where $x_{1}=\left[x_{r}, y_{r}\right]^{T}$, and the two remaining states form $x_{2}$. In other words, the overall state vector $x$ is decomposed into two disjoint subsystems, each of dimension two. Then, system (32) in a decomposed form can be written as

$$
\begin{aligned}
& \dot{x}_{1}=x_{2} \\
& \dot{x}_{2}=u_{2}-u_{1}
\end{aligned}
$$

with $x_{1}, x_{2}, u_{1}, u_{2} \in \mathbb{R}^{2}$.

As level set functions we choose polytopic functions described as intersections of the supporting hyperplanes of the form [19]:

$$
\begin{aligned}
& v_{1}\left(t, x_{1}\right)=\ell_{1}^{T}(t) x_{1}, \\
& v_{2}\left(t, x_{2}\right)=\ell_{2}^{T}(t) x_{2}
\end{aligned}
$$

The protection zone is the target set. Using (28) and the procedure proposed 
in $[3,19]$, we compute

$$
\begin{aligned}
& \frac{d v_{1}}{d t}=\dot{\ell}_{1}^{T} x_{1}+\ell_{1}^{T} x_{2} \\
& \mu_{1}(t)=\max _{u_{1} \in \mathbf{U}_{1}, u_{2} \in \mathbf{U}_{2}}\left\{\ell_{1}^{T}\left(t_{f}\right)\left(u_{2}-u_{1}\right)\right\}, \text { for } \ell_{1}(t) \equiv \ell_{1}\left(t_{f}\right) \\
& \frac{d v_{2}}{d t}=\dot{\ell}_{2}^{T} x_{2}+\ell_{2}^{T}\left(u_{2}-u_{1}\right) \\
& \mu_{2}(t)=\min _{u_{2} \in \mathbf{U}_{2}}\left\{\ell_{2}^{T}\left(t_{f}\right) u_{2}\right\}-\min _{u_{1} \in \mathbf{U}_{1}}\left\{\ell_{2}^{T}\left(t_{f}\right) u_{1}\right\}, \text { for } \ell_{2}(t) \equiv \ell_{2}\left(t_{f}\right)
\end{aligned}
$$

Using equations (31), (34), and (35) we obtain the over-approximation $\Re_{1}$ for $x_{1}$, that is, rectangular relative coordinates $x_{r}$ and $y_{r}$. Notice that in this procedure, the term $\ell_{1}^{T} x_{2}=\ell_{1}^{T}\left(u_{2}-u_{1}\right)$ is treated as an interconnection (that is, perturbation $h_{1}(x)$ in $(27)$ ). By computing the backward reachable approximation $\Re_{2}$ in the $x_{2}$-space, the overall over-approximation is computed as $\Re=\Re_{1} \times \Re_{2}$. In Figure 2 , the inner set is the exact unsafe set in $\left(x_{r}, y_{r}, \psi_{r}\right)$-space, $\psi_{r}$ being the relative heading angle as in Figure 1, and the outer set is the over-approximation obtained by projecting $\Re=\Re_{1} \times \Re_{2}$ into $\left(x_{r}, y_{r}, \psi_{r}\right)$-space. The exact set is computed in [14], using the convergent approximation designed there. This exact computation took approximately 5 minutes on a Sun UltraSparc with 50 grid nodes in each dimension. The over-approximation computation (using MATLAB on a $700 \mathrm{MHz}$ Pentium III PC) took 0.2 seconds (which includes plotting the figure).

If we choose the first three coordinates of the state space vector $x$ as our subsystem, which turns out to be a finer decomposition, and compute the over-approximation in this three dimensional space, we obtain a better over-approximation of the exact set, as shown in Figure 3.

The over-approximation computation plotted in Figure 3 (again, using MATLAB on a $700 \mathrm{MHz}$ Pentium III PC), took 0.31 seconds (including plotting the figure).

Further refinements can be obtained by intersections of various disjoint or overlapping over-approximations, and an efficient methodology for doing this is the subject of our current research. 


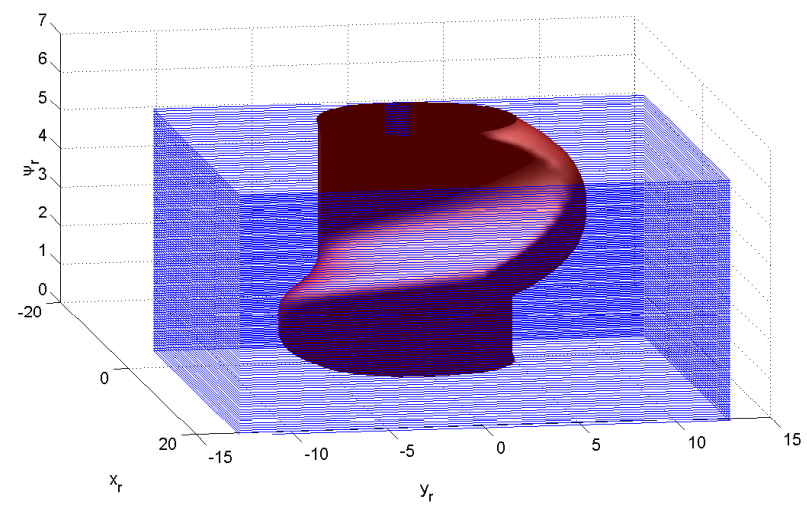

Figure 2: An over-approximation of the exact unsafe set (two 2-dimensional subsystems).

\section{Conclusions}

A method for solving the problem of computation of the backward reachable set, as proposed in this paper, decomposes the problem into a set of smaller dimensional problems on the basis of the decomposition of the overall dynamic system into a set of subsystems. Then the analysis is carried over using a set of subsystem level set functions of which the level sets provide the over-approximations of the projections of the reachable set into subsystem spaces. The method accommodates computation of the reachable sets for two player dynamic games and the application to computation of the unsafe set for the two aircraft collision avoidance problem produced encouraging results. Our future work is to consider computing reachable sets for hybrid systems using subsystem level set functions, and to work on higher dimensional applications.

Finally, it is important to note that the analysis presented in this paper carries over to the computation of the forward reachable set of dynamic systems in an obvious way. 
Comp. of an Over-App. of the Back. Reach. Set using Sub. Level Set Func. 15

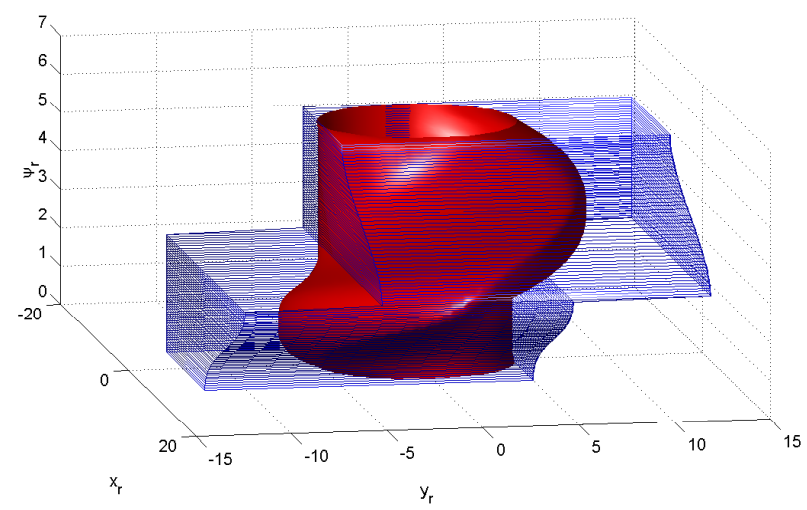

Figure 3: An over-approximation of the exact unsafe set (one 3-dimensional subsystem).

\section{Acknowledgements}

This research is supported by DARPA under the Software Enabled Control Program (AFRL contract F33615-99-C-3014), and by the DoD Multidisciplinary University Research Initiative (MURI) program administered by the Office of Naval Research under Grant N00014-02-1-0720.

\section{References}

[1] M. Bardi and I. Capuzzo-Dolcetta. Optimal Control and Viscosity Solutions of Hamilton-Jacobi-Bellman Equations. Birkhäuser, Boston, MA, 1997.

[2] M. G. Crandall, L. C. Evans, and P.-L. Lions. Some properties of viscosity solutions of Hamilton-Jacobi equations. Transactions of American Mathematical Society, 282(2):487-502, 1984.

[3] I. Hwang, D. M. Stipanović, and C. J. Tomlin. Applications of polytopic approximations of reachable sets to linear dynamic games and a class of nonlinear systems. In Proceedings of the American Control Conference, pages 4613-4619, Denver, June 2003.

[4] M. M. Khrustalev. Exact description of reachable sets and global optimality conditions. Avtomatika i Telemekhanika, (5):62-70, 1988. 
[5] M. M. Khrustalev. Exact description of reachable sets and global optimality conditions II: conditions of global optimality. Avtomatika i Telemekhanika, (7):70-80, 1988.

[6] V. F. Krotov. Global Methods in Optimal Control Theory. Marcel Dekker, Inc, New York, NY, 1996.

[7] A. Kurzhanski and P. Varaiya. Ellipsoidal techniques for reachability analysis. In B. Krogh and N. Lynch, editors, Hybrid Systems: Computation and Control, volume LNCS 1790, pages 202-214. Springer Verlag, 2000.

[8] A. Kurzhanski and P. Varaiya. Dynamic optimization for reachability problems. Journal of Optimization Theory and Applications, 108(2):227-251, 2001.

[9] A. Kurzhanski and P. Varaiya. On reachability under uncertainty. SIAM Journal on Control and Optimization, 41(1):181-216, 2002.

[10] A. Kurzhanski and P. Varaiya. Reachability analysis for uncertain systems-the ellipsoidal technique. Dynamics of Continuous, Discrete \& Impulse Systems, Series B: Applications \& Algorithms, 9(3):347-367, 2002.

[11] G. Leitmann. An Introduction to Optimal Control. Mc-Graw-Hill, New York, NY, 1967.

[12] J. Lygeros, C. J. Tomlin, and S. Sastry. Controllers for reachability specifications for hybrid systems. Automatica, 35(3):349-370, 1999.

[13] I. Mitchell, A. M. Bayen, and C. J. Tomlin. Validating a Hamilton-Jacobi approximation to hybrid system reachable sets. In M. D. D. Benedetto and A. SangiovanniVincentelli, editors, Hybrid Systems: Computation and Control, volume LNCS 2034, pages 418-432. Springer Verlag, 2001.

[14] I. Mitchell, A. M. Bayen, and C. J. Tomlin. Computing reachable sets for continuous dynamic games using level set methods. IEEE Transactions on Automatic Control, 2002. Submitted.

[15] I. Mitchell and C. J. Tomlin. Overapproximating reachable sets by Hamilton-Jacobi projections. Journal of Scientific Computing, special issue dedicated to Stanley Osher on the occasion of his 60th birthday, 2002. Accepted.

[16] S. Sastry. Nonlinear Systems: Analysis, Stability, and Control. Springer Verlag, New York, NY, 1999.

[17] D. M. Stipanović, G. İnalhan, R. Teo, and C. J. Tomlin. Decentralized overlapping control of a formation of unmanned aerial vehicles. In Proceedings of the IEEE Conference on Decision and Control, Las Vegas, Nevada, December 2002. 
Comp. of an Over-App. of the Back. Reach. Set using Sub. Level Set Func. 17

[18] C. J. Tomlin, J. Lygeros, and S. Sastry. A game theoretic approach to controller design for hybrid systems. Proceedings of the IEEE, 88(7):949-970, 2000.

[19] P. Varaiya. Reach set computation using optimal control. In Proceedings of the KIT Workshop on Verification of Hybrid Systems, Grenoble, France, 1998.

[20] T. L. Vincent and W. J. Grantham. Nonlinear and Optimal Control Systems. John Wiley \& Sons, Inc, New York, NY, 1997.

[21] T. L. Vincent and Z. Y. Wu. Estimating projections of the controllable set. Journal of Guidance, Control, and Dynamics, 13(3):572-575, 1990.

[22] R. Vinter. A characterization of the reachable set for nonlinear control systems. SIAM Journal on Control and Optimization, 18(6):599-610, 1980.

[23] D. D. Šiljak. Decentralized Control of Complex Systems. Academic Press, Boston, MA, 1991. 